\title{
Carbon-Nitrogen Bond Formation via the Reaction of Terminal Alkynes with a Dinuclear Side-On Dinitrogen Complex
}

\author{
(Supplementary Material)
}

\author{
Lara Morello, Jason B. Love, Brian O. Patrick, and Michael D. Fryzuk*
}

\section{General Experimental Procedures}

Unless otherwise stated all manipulations were performed under an atmosphere of dry, oxygen-free dinitrogen or argon by means of standard Schlenk or glovebox techniques (Vacuum Atmospheres HE-553-2 glovebox equipped with a $\mathrm{MO}-40-2 \mathrm{H}$ purification system and a $-40^{\circ} \mathrm{C}$ freezer). Argon and nitrogen were dried and deoxygenated by passing the gases through a column containing molecular sieves and $\mathrm{MnO}$. Hexanes and toluene were purchased from Aldrich and dried by passage through a tower of alumina and degassed by passage through a tower of Q-5 catalyst under positive pressure of nitrogen. Anhydrous THF was pre-dried by refluxing over $\mathrm{CaH}_{2}$ and distilled from benzophenone ketyl under argon. Deuterated toluene was refluxed over sodium and potassium alloy under partial pressure, trap-to-trap distilled and freeze-pump-thaw degassed three times. ${ }^{1} \mathrm{H}$ and ${ }^{31} \mathrm{P}\left\{{ }^{1} \mathrm{H}\right\}$ NMR spectra were recorded on either a Bruker AMX-500 instrument operating at 500.1 MHz for ${ }^{1} \mathrm{H}$ spectra or a Bruker AV-300 instrument operating at 300.1 MHz for ${ }^{1} \mathrm{H}$ spectra. ${ }^{1} \mathrm{H}$ NMR spectra were referenced to residual protons in the deuterated solvent: $\mathrm{C}_{6} \mathrm{D}_{5} \mathrm{H}$ (7.15 ppm) and $\mathrm{C}_{7} \mathrm{D}_{7} \mathrm{H}$ (2.09 ppm). ${ }^{31} \mathrm{P}\left\{{ }^{1} \mathrm{H}\right\}$ NMR spectra were referenced to external $\mathrm{P}(\mathrm{OMe})_{3}$ (141.0 ppm with respect to $85 \% \mathrm{H}_{3} \mathrm{PO}_{4}$ at $0.0 \mathrm{ppm}$ ) or to a known impurity within the sample (36.8 ppm singlet, $\left[\mathrm{P}_{2} \mathrm{~N}_{2}\right] \mathrm{H}_{2}$ ).

Microanalyses (C, H, N) were performed by Mr. P. Borda or Mr. M. Lakha and mass spectroscopy (EI low res.) was performed by Mr. M. Lapawa; all of the University of British Columbia Department of Chemistry.

\section{Materials}

Compound 1, $\left(\left[\mathrm{P}_{2} \mathrm{~N}_{2}\right] \mathrm{Zr}\right)_{2}\left(\mu-\eta^{2}: \eta^{2}-\mathrm{N}_{2}\right)$, was synthesized according to a literature procedure. ${ }^{1}$ Phenylacetylene was purchased from Aldrich. The para-substituted phenylacetylenes, 4-ethynyltoluene (OR p-tolylacetylene) and (p-tert-butylphenyl)acetylene, were purchased from 
GFS Chemicals. All acetylenes were distilled, freeze-pump-thaw degassed three times prior to use and stored over molecular sieves in the dark.

\section{Synthesis and Characterization of $\left(\left[\mathrm{P}_{2} \mathrm{~N}_{2}\right] \mathrm{Zr}\right)_{2}\left(\mu_{2}-\mathrm{N}_{2} \mathrm{CHCHPh}\right)\left(\mu_{2}-\mathrm{CCPh}\right), 2$}

An excess of phenylacetylene (280 $\mathrm{mg}, 2.74 \mathrm{mmol}$ ) was added to a stirred solution of $\left(\left[\mathrm{P}_{2} \mathrm{~N}_{2}\right] \mathrm{Zr}\right)_{2}\left(\mu-\eta^{2}: \eta^{2}-\mathrm{N}_{2}\right)$ (250mg, $\left.0.20 \mathrm{mmol}\right)$ in toluene $(10 \mathrm{~mL})$ at room temperature. The mixture was stirred for $48 \mathrm{~h}$ (initially the color became orange with an orange precipitate forming after prolonged reaction), after which the solvents were evaporated and the orange residue extracted into a minimum of hot THF (ca. $5 \mathrm{~mL}$ ). Layering the cooled THF solution with hexanes (30 $\mathrm{mL}$ ) caused star-like clusters of $\mathbf{2}$ to form, which were isolated by decanting the liquors, washing with hexanes (2 x $2 \mathrm{~mL})$ and drying under vacuum. Yield 200mg, 69\%.

Anal. Calcd for $\mathrm{C}_{64} \mathrm{H}_{96} \mathrm{~N}_{6} \mathrm{P}_{4} \mathrm{Si}_{8} \mathrm{Zr}_{2}$ (hexane) 0.5 : C, 53.66; H, 7.08; N, 5.36. Found: C, 53.59; H, 6.92; N, 5.22.

${ }^{1} \mathbf{H}$ NMR $\left(\mathbf{C}_{\mathbf{4}} \mathbf{D}_{\mathbf{8}} \mathbf{O}, 500 \mathbf{M H z}, 298 \mathbf{~ K}\right): \delta_{\mathrm{H}}-0.37$ (s, 6H, ring SiMe $)$ ), -0.25 (s, 6H, ring $\mathrm{SiMe}_{2}$ ), 0.01 (s, 6H, ring $\mathrm{SiMe}_{2}$ ), 0.02 (s, 6H, ring $\mathrm{SiMe}_{2}$ ), 0.12 (s, 6H, ring $\mathrm{SiMe}_{2}$ ), 0.14 (s, 6H, ring $\mathrm{SiMe}_{2}$ ), 0.18 (s, 6H, ring $\mathrm{SiMe}_{2}$ ), 0.34 (s, 6H, ring $\mathrm{SiMe}_{2}$ ), 0.83 (br.m, 8H, ring $\mathrm{CH}_{2}$ ), 1.26 (br.m, 8H, ring $\mathrm{CH}_{2}$ ), 6.06 (d, $1 \mathrm{H}, \mathrm{J}_{\mathrm{HH}} 13.8 \mathrm{~Hz}$, NCHCHPh), 6.36 (d, 2H, $7.25 \mathrm{~Hz}$, phenyl), 6.75 (t, 2H, $\mathrm{J}_{\mathrm{HH}} 7.25 \mathrm{~Hz}$, phenyl), 6.86 (t, 2H, $\mathrm{J}_{\mathrm{HH}} 7.85 \mathrm{~Hz}$, phenyl), 7.30 (br.m’s, 18H, phenyl), 7.44 (dd, 2H, $\mathrm{J}_{\mathrm{HH}} 8.10 \& 7.85 \mathrm{~Hz}$, phenyl), 7.55 (d, 1H, $\mathrm{J}_{\mathrm{HH}} 13.9 \mathrm{~Hz}$, NCHCHPh), 7.62 (br.s, 4H, o-H phenyl).

${ }^{1} \mathbf{H}$ NMR $\left(\mathbf{C}_{4} \mathbf{D}_{\mathbf{8}} \mathbf{O}, 500 \mathrm{MHz}, 230 \mathrm{~K}\right): \delta_{\mathrm{H}}-0.64$ (s, 3H, ring $\mathrm{SiMe}_{2}$ ), -0.49 (s, 3H, ring $\mathrm{SiMe}_{2}$ ), 0.40 (s, 3H, ring $\mathrm{SiMe}_{2}$ ), -0.08 (s, 3H, ring $\mathrm{SiMe}_{2}$ ), -0.07 (s, 3H, ring $\mathrm{SiMe}_{2}$ ), -0.01 (s, 3H, ring $\mathrm{SiMe}_{2}$ ), 0.02 (s, 3H, ring $\mathrm{SiMe}_{2}$ ), 0.06 (s, 3H, ring $\mathrm{SiMe}_{2}$ ), 0.08 (s, 3H, ring $\mathrm{SiMe}_{2}$ ), 0.09 (s, 3H, ring $\mathrm{SiMe}_{2}$ ), 0.11 (s, 3H, ring $\mathrm{SiMe}_{2}$ ), 0.14 (s, 3H, ring SiMe 2 ), 0.16 (s, 3H, ring $\mathrm{SiMe}_{2}$ ), 0.24 (s, 3H, ring $\mathrm{SiMe}_{2}$ ), 0.35 (s, 3H, ring $\mathrm{SiMe}_{2}$ ), 0.39 (s, 3H, ring $\mathrm{SiMe}_{2}$ ), 1.80 to 0.60 (overlapping multiplets, 16H, ring $\mathrm{CH}_{2}$ ), 5.99 (d, 1H, $\left.\mathrm{J}_{\mathrm{HH}} 13.7 \mathrm{~Hz}, \mathrm{NCHCHPh}\right), 6.67$ (m, 2H, phenyl), 6.76 (m, 2H, phenyl), 6.84 (br.s, 2H, phenyl), 7.54 to 7.10 (overlapping multiplets, 20H, phenyl), 7.56 (d,

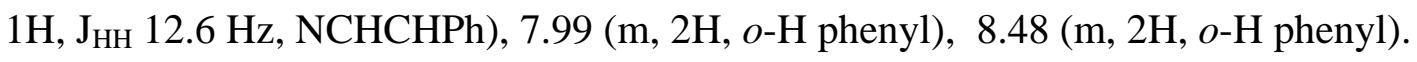

${ }^{31} \mathbf{P}\left\{{ }^{1} \mathrm{H}\right\}$ NMR ( $\left.\mathrm{C}_{4} \mathbf{D}_{8} \mathrm{O}, 121 \mathrm{MHz}, 298 \mathrm{~K}\right): \delta_{\mathrm{P}}-14.3$ (br.s); ${ }^{31} \mathbf{P}\left\{{ }^{1} \mathrm{H}\right\}$ NMR $\left(\mathrm{C}_{4} \mathrm{D}_{8} \mathrm{O}, 121 \mathrm{MHz}, 215\right.$ K): $\delta_{P}-16.7$ (d, JPP $75.4 \mathrm{~Hz}$ ), -16.0 (d, JPP $68.5 \mathrm{~Hz}$ ), -10.2 (d, JPP $68.5 \mathrm{~Hz}$ ), -9.77 (d, JPP $75.4 \mathrm{~Hz}$ ). 


\section{Synthesis and Characterization of $\left(\left[\mathrm{P}_{2} \mathrm{~N}_{2}\right] \mathrm{Zr}\right)_{2}\left[\mu_{2}-\mathrm{N}_{2} \mathrm{CHCHC}_{6} \mathrm{H}_{4}\left(4-\mathrm{CH}_{3}\right)\right]\left[\mu_{2}-\mathrm{CCC}_{6} \mathrm{H}_{4}(4-\right.$}

$\left.\mathrm{CH}_{3}\right)$ ], 3

A $0.06 \mathrm{M}$ solution of ( $p$-methyl)phenylacetylene was quantitatively prepared in toluene. A slight excess of $p$-tolylacetylene $(0.06 \mathrm{M}, 8 \mathrm{~mL}, 0.48 \mathrm{mmol})$ was added to a stirred solution of $\left(\left[\mathrm{P}_{2} \mathrm{~N}_{2}\right] \mathrm{Zr}\right)_{2}\left(\mu-\eta^{2}: \eta^{2}-\mathrm{N}_{2}\right)(250 \mathrm{mg}, 0.20 \mathrm{mmol})$ in toluene $(10 \mathrm{~mL})$ at room temperature. The mixture was not stirred and a color change from blue/green to orange was observed over the course of a week. The solution was concentrated to a brown oil and hexanes ( $\sim 5 \mathrm{~mL})$ were added. Orange crystalline platelets of $\mathbf{3}$ were deposited in situ from the slow evaporation of the toluene/hexanes solution. Yield $133 \mathrm{mg}, 45 \%$.

Anal. Calcd. for $\mathrm{C}_{66} \mathrm{H}_{100} \mathrm{~N}_{6} \mathrm{P}_{4} \mathrm{Si}_{8} \mathrm{Zr}_{2}$ : C, 52.55; H, 6.68; N, 5.57. Found: C, 52.95; H, 6.87; N, 5.74. MS EI Low res. $\left(\mathrm{M}^{+}\right) \mathrm{m} / \mathrm{z}: 1509$.

${ }^{1} \mathbf{H}$ NMR ( $\mathbf{C}_{7} \mathbf{D}_{\mathbf{8}}, 300 \mathrm{MHz}, 300 \mathrm{~K}$ ): $\delta_{\mathrm{H}}-0.06$ (s, 6H, ring SiMe $)$ ), 0.04 (br.s, 6H, ring $\mathrm{SiMe}_{2}$ ), 0.09 (s, 3H, ring $\mathrm{SiMe}_{2}$ ), 0.13 (s, 3H, ring $\mathrm{SiMe}_{2}$ ), 0.23 (s, 6H, ring $\mathrm{SiMe}_{2}$ ), 0.26 to 0.27 (overlapping singlets, $6 \mathrm{H}$, ring $\mathrm{SiMe}_{2}$ ), 0.34 (s, $6 \mathrm{H}$, ring $\mathrm{SiMe}_{2}$ ), 0.39 to 0.41 (overlapping singlets, $12 \mathrm{H}$, ring SiMe ), 0.74 to 1.77 (br.m, 16H, ring $\mathrm{CH}_{2}$ ), 1.97 (s, 3H, $\mathrm{PhCH}_{3}$ ), 2.14 (s, 3H, $\mathrm{PhCH}_{3}$ ), 6.34 (d, $1 \mathrm{H}$, $\mathrm{J}_{\mathrm{HH}} 14.0 \mathrm{~Hz}, \mathrm{NCHCHPh}(\mathrm{Me})$ ), 6.68 (d, 2H, $\mathrm{J}_{\mathrm{HH}} 7.9 \mathrm{~Hz}$, phenyl), 6.86 (d, 2H, J $\mathrm{J}_{\mathrm{HH}} .9 \mathrm{~Hz}$, phenyl), 6.91 (d, 2H, $\mathrm{J}_{\mathrm{HH}} 8.3 \mathrm{~Hz}$, phenyl), 7.09 to 7.33 (br.m, 16H, phenyl), 7.53 (d, 2H, $\mathrm{J}_{\mathrm{HH}} 8.3 \mathrm{~Hz}$, phenyl), 7.59 to 7.78 (br.m, 5H, phenyl and NCHCHPh(Me)).

${ }^{1} \mathbf{H}$ NMR (C $\mathbf{C}_{7} \mathbf{D}_{\mathbf{8}}, 300 \mathrm{MHz}, 240 \mathrm{~K}$ ): $\delta_{\mathrm{H}}-0.18$ (s, 3H, ring SiMe $)$ ), -0.02 (s, 6H, ring $\mathrm{SiMe}_{2}$ ), 0.10 (s, 3H, ring $\mathrm{SiMe}_{2}$ ), 0.17 (s, 3H, ring $\mathrm{SiMe}_{2}$ ), 0.26 (s, 3H, ring $\mathrm{SiMe}_{2}$ ), 0.32 (overlapping singlets, 12H, ring $\mathrm{SiMe}_{2}$ ), 0.36 (s, $3 \mathrm{H}$, ring $\mathrm{SiMe}_{2}$ ), 0.39 (s, 3H, ring $\mathrm{SiMe}_{2}$ ), 0.45 (overlapping singlets, 6H, ring $\mathrm{SiMe}_{2}$ ), 0.58 (s, 3H, ring $\mathrm{SiMe}_{2}$ ), 0.73 (s, 3H, ring $\mathrm{SiMe}_{2}$ ), 0.81 to 1.64 (br.m, 16H, ring

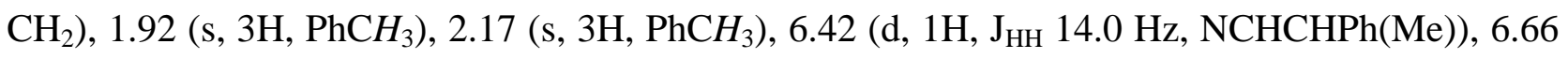
(d, 2H, $\mathrm{J}_{\mathrm{HH}} 7.9 \mathrm{~Hz}$, phenyl), 6.81 (d, 2H, $\mathrm{J}_{\mathrm{HH}} 7.9 \mathrm{~Hz}$, phenyl), 6.88 (br. m, 2H, phenyl), 7.14 to 7.25 (br. m, 10H, phenyl), 7.34 to 7.46 (br. m, 6H, phenyl), 7.59 (d, 2H, J 7.9 Hz, phenyl), 7.77 (d, 1H, J $\mathrm{J}_{\mathrm{HH}} 14.0 \mathrm{~Hz}, \mathrm{NCHCHPh}(\mathrm{Me})$ ), 7.90 (m, 2H, phenyl), 8.37 (m, 2H, phenyl).

${ }^{31} \mathbf{P}\left\{{ }^{1} \mathbf{H}\right\}$ NMR (C $\left.7 \mathbf{D}_{8}, 121 \mathrm{MHz}, 300 \mathrm{~K}\right): \delta_{\mathrm{P}}-14.4$ (br.s); ${ }^{31} \mathbf{P}\left\{{ }^{1} \mathbf{H}\right\}$ NMR $\left(C_{7} \mathbf{D}_{8}, 121 \mathrm{MHz}, 240\right.$ K): $\delta_{\mathrm{P}}-16.4\left(\mathrm{~d}, \mathrm{~J}_{\mathrm{PP}} 74.3 \mathrm{~Hz}\right.$ ), -16.0 (d, JPP $66.8 \mathrm{~Hz}$ ), -10.3 (d, JPP $66.8 \mathrm{~Hz}$ ), -9.8 (d, JPP $74.3 \mathrm{~Hz}$ ). 


\section{Synthesis and Characterization of $\left(\left[\mathrm{P}_{2} \mathrm{~N}_{2}\right] \mathrm{Zr}\right)_{2}\left[\mu_{2}-\mathrm{N}_{2} \mathrm{CHCHC}_{6} \mathrm{H}_{4}\left(4-\mathrm{C}\left(\mathrm{CH}_{3}\right)_{3}\right]\left[\mu_{2}-\mathrm{CCC}_{6} \mathrm{H}_{4}(4-\right.\right.$}

$\left.\mathrm{C}\left(\mathrm{CH}_{3}\right)_{3}\right], 4$

A slight excess of (p-tert-butylphenyl)acetylene (198mg, $1.25 \mathrm{mmol}$ ) was added to a stirred solution of $\left(\left[\mathrm{P}_{2} \mathrm{~N}_{2}\right] \mathrm{Zr}\right)_{2}\left(\mu-\eta^{2}: \eta^{2}-\mathrm{N}_{2}\right)(500 \mathrm{mg}, 0.40 \mathrm{mmol})$ in toluene $(10 \mathrm{~mL})$ at room temperature. The mixture was left undisturbed and a color change of blue/green to orange was observed over

the course of one week. Slow evaporation yielded orange needle-like crystals of 4 . Yield 200mg, $40 \%$.

Anal. Calcd. for $\mathrm{C}_{72} \mathrm{H}_{112} \mathrm{~N}_{6} \mathrm{P}_{4} \mathrm{Si}_{8} \mathrm{Zr}_{2}$ : C, 54.29; H, 7.09; N, 5.28. Found: C, 53.78; H, 7.18; N, 5.52. MS EI Low res. $\left(\mathrm{M}^{+}\right) \mathrm{m} / \mathrm{z}: 1592$.

${ }^{1} \mathbf{H}$ NMR ( $\mathbf{C}_{7} \mathbf{D}_{\mathbf{8}}, 300 \mathrm{MHz}, 299$ K): $\delta_{\mathrm{H}}-0.06$ (s, 6H, ring SiMe 2 ), 0.04 (br.s, 6H, ring $\mathrm{SiMe}_{2}$ ), 0.23 to 0.40 (overlapping br. singlets, $30 \mathrm{H}$, ring $\mathrm{SiMe}_{2}$ ), 0.66 (br.s, $6 \mathrm{H}$, ring $\mathrm{SiMe}_{2}$ ), 0.89 (br.m, 8H, ring $\mathrm{CH}_{2}$ ), 1.09 (s, 9H, ${ }^{\mathrm{t}} \mathrm{Bu}$ ), 1.23 (br.m, 8H, ring $\mathrm{CH}_{2}$ ), 1.26 (s, 9H, ${ }^{\mathrm{t}} \mathrm{Bu}$ ), 6.35 (d, $1 \mathrm{H}, \mathrm{J}_{\mathrm{HH}} 13.9$ $\mathrm{Hz}, \mathrm{NCHCHPh}\left({ }^{\mathrm{t}} \mathrm{Bu}\right)$ ), 6.78 (d, 2H, $\mathrm{J}_{\mathrm{HH}} 8.5 \mathrm{~Hz}$, phenyl), 6.92 to 7.31 (overlapping br. multiplets, 20H, phenyl), 7.37 (d, 2H, $\mathrm{J}_{\mathrm{HH}} 8.5 \mathrm{~Hz}$, phenyl), 7.59 (d, 2H, $\mathrm{J}_{\mathrm{HH}} 8.5 \mathrm{~Hz}$, phenyl), 7.61 to 7.79 (br. $\mathrm{m}, 3 \mathrm{H}$, phenyl and $\left.\mathrm{NCHCHPh}\left({ }^{\mathrm{t}} \mathrm{Bu}\right)\right)$.

${ }^{1} \mathbf{H}$ NMR ( $\mathbf{C}_{7} \mathbf{D}_{\mathbf{8}}, 300 \mathrm{MHz}, 235 \mathrm{~K}$ ): $\delta_{\mathrm{H}}-0.13$ (s, 3H, ring SiMe 2 ), -0.06 (s, 3H, ring $\mathrm{SiMe}_{2}$ ), 0.00 (s, 3H, ring $\mathrm{SiMe}_{2}$ ), 0.21 (s, 3H, ring $\mathrm{SiMe}_{2}$ ), 0.23 (s, 3H, ring $\mathrm{SiMe}_{2}$ ), 0.30 (s, 3H, ring $\mathrm{SiMe}_{2}$ ), 0.35 (br. s, 15H, ring $\mathrm{SiMe}_{2}$ ), 0.47 (br. s, 6H, ring $\mathrm{SiMe}_{2}$ ), 0.54 (s, 3H, ring $\mathrm{SiMe}_{2}$ ), 0.58 (s, 3H, ring $\mathrm{SiMe}_{2}$ ), 0.72 (s, 3H, ring $\mathrm{SiMe}_{2}$ ), 0.87 to 0.96 (overlapping multiplets, $8 \mathrm{H}$, ring $\mathrm{CH}_{2}$ ), 1.06 (s, $9 \mathrm{H},{ }^{\mathrm{t}} \mathrm{Bu}$ ), 1.15 to 1.26 (overlapping multiplets, $8 \mathrm{H}$, ring $\mathrm{CH}_{2}$ ), 1.30 (s, $9 \mathrm{H},{ }^{\mathrm{t}} \mathrm{Bu}$ ), 6.46 (d, $1 \mathrm{H}, \mathrm{J}_{\mathrm{HH}}$ $13.9 \mathrm{~Hz}, \mathrm{NCHCHPh}\left({ }^{\mathrm{B} u}\right.$ )), 6.82 (br. d, 2H, phenyl), 6.90 to 7.45 (overlapping multiplets, $20 \mathrm{H}$,

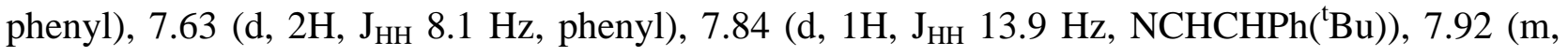
$2 \mathrm{H}$, phenyl), 8.38 ( $\mathrm{m}, 2 \mathrm{H}$, phenyl).

${ }^{31} \mathbf{P}\left\{{ }^{1} \mathbf{H}\right\}$ NMR $\left(C_{7} \mathbf{D}_{8}, 121 \mathrm{MHz}, 299 \mathrm{~K}\right): \delta_{\mathrm{P}}-14.3$ (br.s); ${ }^{31} \mathbf{P}\left\{{ }^{1} \mathbf{H}\right\}$ NMR $\left(C_{7} \mathbf{D}_{8}, 121 \mathrm{MHz}, 235\right.$ K): $\delta_{\mathrm{P}}-15.5$ (d, JPP $74.1 \mathrm{~Hz}$ ), -15.1 (d, JPP $65.6 \mathrm{~Hz}$ ), -10.8 (d, JPP $65.6 \mathrm{~Hz}$ ), -10.4 (d, JPP $74.1 \mathrm{~Hz}$ ).

\section{Kinetic Isotope Experiments}

One sample of $\left(\left[\mathrm{P}_{2} \mathrm{~N}_{2}\right] \mathrm{Zr}\right)_{2}\left(\mu_{2}-\eta^{2}-\mathrm{N}_{2}\right)(11.9 \mathrm{mg}, 0.00933 \mathrm{mmol})$ was weighed out into a vial and dissolved with $\sim 0.6 \mathrm{~mL}$ of $\mathrm{d}_{8}$-toluene. The dark teal coloured solution was transferred to a $1.00 \mathrm{~mL}$ volumetric "flask" and $\mathrm{d}_{8}$-toluene was used to make the sample up to volume. A $1.00 \mathrm{~mL}$ syringe was used to transfer $0.40 \mathrm{~mL}$ of the solution into each of two NMR tubes. A sealed 
capillary tube containing $30 \mu \mathrm{L}$ of NEAT PEt 3 was added to each of the NMR tubes. A $10 \mu \mathrm{L}$ syringe was washed with PhCCH (or PhCCD) prior to delivering $1 \mu \mathrm{L}$ ( 2.4 eq.) to one of the NMR samples.

\begin{tabular}{cccccc}
\hline $\begin{array}{c}\text { Sample } \\
\text { mmol of } \mathbf{1}\end{array}$ & $\begin{array}{c}\text { Volume mL } \\
\mathrm{d}_{8} \text {-toluene }\end{array}$ & $\begin{array}{c}\text { Concentration } \\
(\mathrm{mol} / \mathrm{L}) \text { of } \mathbf{1}\end{array}$ & $\begin{array}{c}\text { mmol of } \\
\text { PhCCH/D }\end{array}$ & $\begin{array}{c}\text { or }[\mathrm{PhCCH}] \\
(\mathrm{mol} / \mathrm{L})\end{array}$ \\
\hline $1 . \mathbf{1}+2 \mathrm{PhCCH}$ & 0.00373 & 0.40 & 0.00933 & 0.00911 & 0.0228 \\
$2 . \mathbf{1}+2$ PhCCD & 0.00373 & 0.40 & 0.00933 & 0.00910 & 0.0228 \\
\hline
\end{tabular}

The samples were mixed at the same time and monitored by ${ }^{31} \mathrm{P}\left\{{ }^{1} \mathrm{H}\right\}$ NMR every 10 minutes over the course of 3 hours. A spectrum, for each sample, was also obtained after 5 hours and again after 4 weeks. The resonance due to the $\mathrm{Zr}-\mathrm{N}_{2}$ complex $(\delta-16.3$, s) was quantified by integration with respect to the internal standard, $\operatorname{PEt}_{3}(\delta-62.5, \mathrm{~s})$, which was assigned a value of 1000 in all spectra. The 'peaks' were integrated over the same range for each spectrum. Plotting the decrease in the concentration of $\mathbf{1}$ in both experiments gave virtually identical values over the period indicated; the data are estimated to be accurate to $\pm 10 \%$.

\section{X-Ray Crystallography}

A summary of all crystal data collection and refinement for compounds $\mathbf{2}, \mathbf{3}$, and $\mathbf{4}$ is given in Table 1. Crystal structure data CIF files are available. Tables 2, 3 and 4 contain selected bond lengths and angles for compounds $\mathbf{2 ,} 3$ and 4, respectively.

Measurements for compound 2 were made on a Rigaku AFC6S diffractometer with graphite monochromated Mo-K $\alpha$ radiation. The structure of 2 was solved by heavy-atom Patterson methods ${ }^{2}$ and expanded using Fourier techniques. ${ }^{3}$ There is one-half of a 'hexane' molecule per metal complex in the lattice, located near a center of symmetry. The hexane carbon atoms were refined with isotropic thermal parameters and all remaining non-hydrogen atoms were refined anisotropically. Hydrogen atoms were fixed in calculated positions with $\mathrm{C}-\mathrm{H}=0.98 \AA$.

All measurements for compounds $\mathbf{3}$ and $\mathbf{4}$ were made on an ADSC CCD area detector coupled with a Rigaku AFC7 diffractometer with graphite monochromated Mo-K $\alpha$ radiation. Data were collected and processed using the $\mathrm{d}^{*}$ TREK program. ${ }^{4}$ The structures of $\mathbf{3}$ and $\mathbf{4}$ were solved by direct methods ${ }^{5,6}$ and expanded using Fourier techniques. ${ }^{3}$ Hydrogen atoms were included but 
not refined. Non-hydrogen atoms were refined anisotropically. Compound $\mathbf{3}$ crystallizes with one half molecule of hexane in the asymmetric unit. This solvent molecule was found to be disordered in two orientations with equal populations. Its atoms were refined anisotropically, however hydrogen atoms were not included in the model. Compound $\mathbf{4}$ crystallizes with two molecules of toluene in the asymmetric unit.

Table 1. Summary of Crystal Data Collection and Refinement for Compounds 2, 3 and 4

\begin{tabular}{|c|c|c|c|}
\hline & $2^{a}$ & $3^{b}$ & $4^{b}$ \\
\hline Empirical Formula & $\mathrm{Zr}_{2} \mathrm{P}_{4} \mathrm{Si}_{8} \mathrm{~N}_{6} \mathrm{C}_{67} \mathrm{H}_{103}$ & $\mathrm{Zr}_{2} \mathrm{P}_{4} \mathrm{Si}_{8} \mathrm{~N}_{6} \mathrm{C}_{69} \mathrm{H}_{107}$ & $\mathrm{Zr}_{2} \mathrm{P}_{4} \mathrm{Si}_{8} \mathrm{~N}_{6} \mathrm{C}_{86} \mathrm{H}_{128}$ \\
\hline $\mathrm{FW}$ & 1523.61 & 1551.66 & 1777.02 \\
\hline Colour, Habit & orange, prism & orange, platelet & clear, needle \\
\hline Crystal size, mm & $0.20 \times 0.37 \times 0.45$ & $0.40 \times 0.20 \times 0.07$ & $0.50 \times 0.10 \times 0.10$ \\
\hline Crystal system & triclinic & triclinic & monoclinic \\
\hline Space group & $\bar{P} 1(\# 2)$ & $\bar{P} 1(\# 2)$ & $\mathrm{P} 2_{1} / \mathrm{a}(\# 14)$ \\
\hline $\mathrm{a}, \AA$ & 13.592(1) & $12.5329(7)$ & 27.261(1) \\
\hline $\mathrm{b}, \AA$ & $24.687(2)$ & 13.3989(5) & $12.7830(4)$ \\
\hline c, $\AA$ & $12.543(1)$ & $25.997(2)$ & $28.343(1)$ \\
\hline$\alpha, \operatorname{deg}$ & 100.987(7) & $91.876(2)$ & \\
\hline$\beta$, deg & $107.008(6)$ & $103.279(1)$ & $108.920(2)$ \\
\hline$\gamma$, deg & $81.744(7)$ & $107.544(2)$ & \\
\hline $\mathrm{V}, \AA^{3}$ & $3933.6(6)$ & $4026.6(4)$ & $9343.2(6)$ \\
\hline $\mathrm{Z}$ & 2 & 2 & 4 \\
\hline $\mathrm{T},{ }^{\circ} \mathrm{C}$ & $21 \pm 1$ & $-100 \pm 1$ & $-100 \pm 1$ \\
\hline$\rho_{\text {calc }}, \mathrm{g} / \mathrm{cm}^{3}$ & 1.286 & 1.280 & 1.263 \\
\hline $\mathrm{F}_{000}$ & 1598.00 & 1630.00 & 3752.00 \\
\hline$\mu(\mathrm{MoK} \alpha), \mathrm{cm}^{-1}$ & 5.09 & 4.98 & 4.38 \\
\hline correction factors & $0.8790-1.0000$ & $0.7231-1.0000$ & $0.8607-1.0000$ \\
\hline $2 \theta_{\max }$, deg & 60 & 56 & 55.7 \\
\hline total no. of reflns & 23796 & 32629 & 87468 \\
\hline no. of unique reflns & 22902 & 15659 & 21250 \\
\hline $\mathrm{R}_{\text {int }}$ & 0.035 & 0.046 & 0.121 \\
\hline $\begin{array}{l}\text { no. observations (I } \\
>2 \sigma(\mathrm{I}))\end{array}$ & 13212 & 12548 & 11779 \\
\hline no. of variables & 785 & 824 & 979 \\
\hline $\mathrm{R} 1\left(\mathrm{~F}^{2}, \mathrm{I}>2 \sigma(\mathrm{I})\right)$ & 0.035 & 0.055 & 0.050 \\
\hline wR2 (F ${ }^{2}$, all data) & 0.102 & 0.136 & 0.103 \\
\hline Gof & 0.97 & 1.12 & 0.88 \\
\hline
\end{tabular}

${ }^{\mathrm{a}}$ Rigaku AFC6S diffractometer, ${ }^{\mathrm{b}}$ Rigaku/ADSC CCD diffractometer

$\mathrm{R} 1=\Sigma|| \mathrm{F}_{\mathrm{o}}|-| \mathrm{F}_{\mathrm{c}}|| / \Sigma\left|\mathrm{F}_{\mathrm{o}}\right| ; \mathrm{wR} 2=\left(\sum \mathrm{w}\left(\left|\mathrm{F}_{\mathrm{o}}{ }^{2}\right|-\left|\mathrm{F}_{\mathrm{c}}{ }^{2}\right|\right)^{2} / \Sigma \mathrm{w}\left|\mathrm{F}_{\mathrm{o}}{ }^{2}\right|^{2}\right)^{1 / 2}$. 


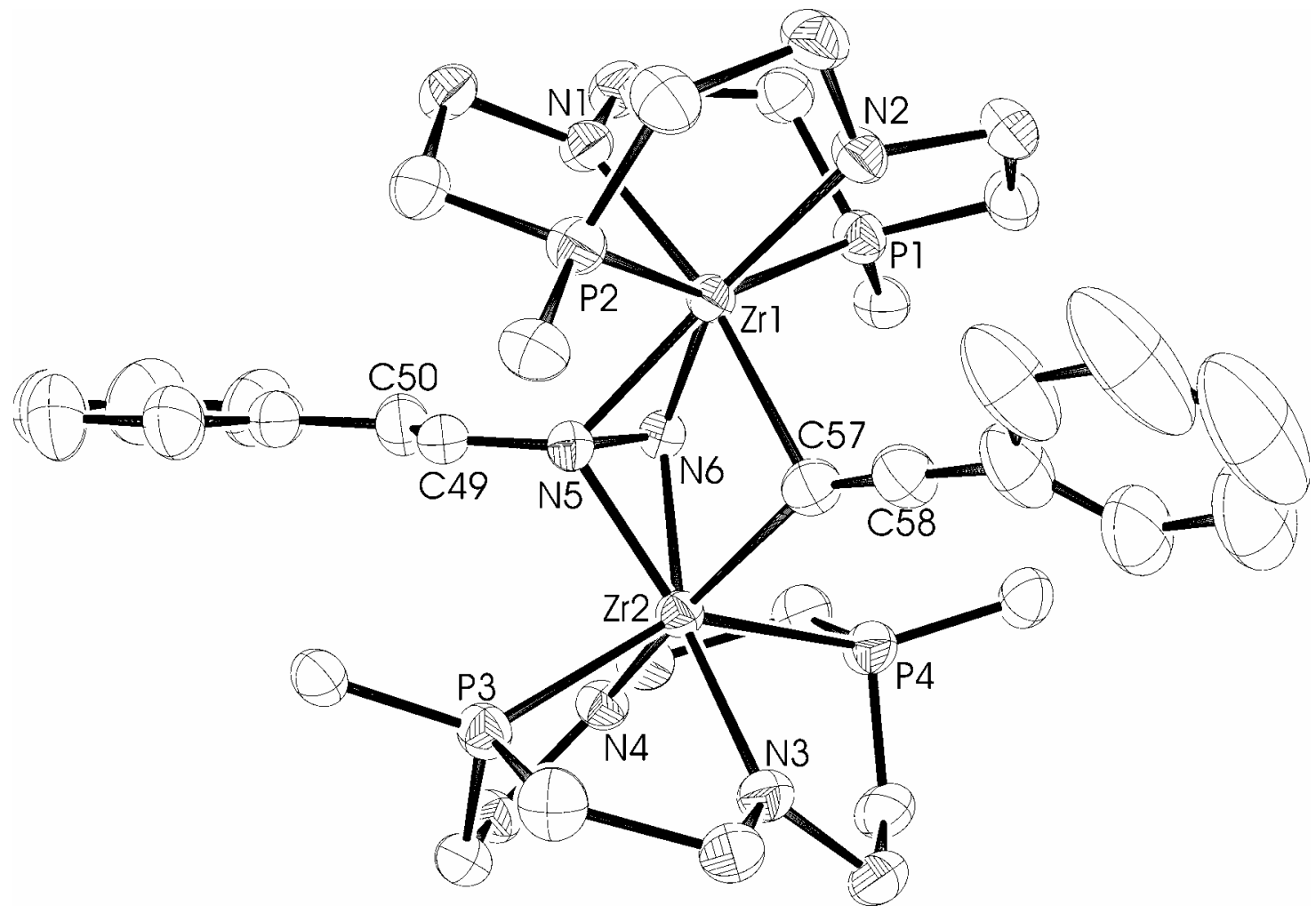

Figure 1: Molecular structure (ORTEP) of ([ $\left.\left.\mathrm{P}_{2} \mathrm{~N}_{2}\right] \mathrm{Zr}\right)_{2}\left(\mu-\mathrm{N}_{2} \mathrm{CHCHPh}\right)(\mu-\mathrm{CCPh}), 2$, determined by X-ray crystallography. Ellipsoids drawn at $50 \%$ probability level, silylmethyls are omitted for clarity and only ipso carbons of phenyl rings are shown.

Table 2: Selected Bond Lengths $(\AA)$ and Angles (deg) in $\left(\left[\mathrm{P}_{2} \mathrm{~N}_{2}\right] \mathrm{Zr}\right)_{2}\left(\mu-\mathrm{N}_{2} \mathrm{CHCHPh}\right)(\mu-\mathrm{CCPh})(2)$

$\begin{array}{llll}\mathrm{N}(5)-\mathrm{N}(6) & 1.445(2) & \mathrm{N}(3)-\operatorname{Zr}(2)-\mathrm{N}(4) & 95.35(7) \\ \mathrm{N}(5)-\mathrm{C}(49) & 1.414(3) & \mathrm{N}(1)-\operatorname{Zr}(1)-\mathrm{N}(2) & 91.68(8) \\ \mathrm{C}(49)-\mathrm{C}(50) & 1.313(4) & \mathrm{P}(1)-\operatorname{Zr}(1)-\mathrm{P}(2) & 153.68(2) \\ \mathrm{C}(57)-\mathrm{C}(58) & 1.208(3) & \mathrm{P}(3)-\operatorname{Zr}(2)-\mathrm{P}(4) & 145.88(2) \\ \operatorname{Zr}(1)-\mathrm{C}(57) & 2.477(2) & \operatorname{Zr}(1)-\mathrm{C}(57)-\operatorname{Zr}(2) & 87.72(8) \\ \operatorname{Zr}(2)-\mathrm{C}(57) & 2.449(3) & \operatorname{Zr}(1)-\mathrm{N}(5)-\operatorname{Zr}(2) & 103.44(8) \\ \operatorname{Zr}(1)-\mathrm{N}(5) & 2.200(2) & \operatorname{Zr}(1)-\mathrm{N}(6)-\operatorname{Zr}(2) & 107.11(8) \\ \operatorname{Zr}(1)-\mathrm{N}(6) & 2.089(2) & & \\ \operatorname{Zr}(2)-\mathrm{N}(5) & 2.149(2) & & \\ \operatorname{Zr}(2)-\mathrm{N}(6) & 2.154(2) & & \\ \operatorname{Zr}(1)-\mathrm{P}(1) & 2.7860(7) & \operatorname{Zr}(2)-\mathrm{P}(3) & 2.7659(7) \\ \operatorname{Zr}(1)-\mathrm{P}(2) & 2.7645(7) & \operatorname{Zr}(2)-\mathrm{P}(4) & 2.7450(7) \\ \operatorname{Zr}(1)-\mathrm{N}(1) & 2.198(2) & \operatorname{Zr}(2)-\mathrm{N}(3) & 2.304(2) \\ \operatorname{Zr}(1)-\mathrm{N}(2) & 2.303(2) & \operatorname{Zr}(2)-\mathrm{N}(4) & \end{array}$




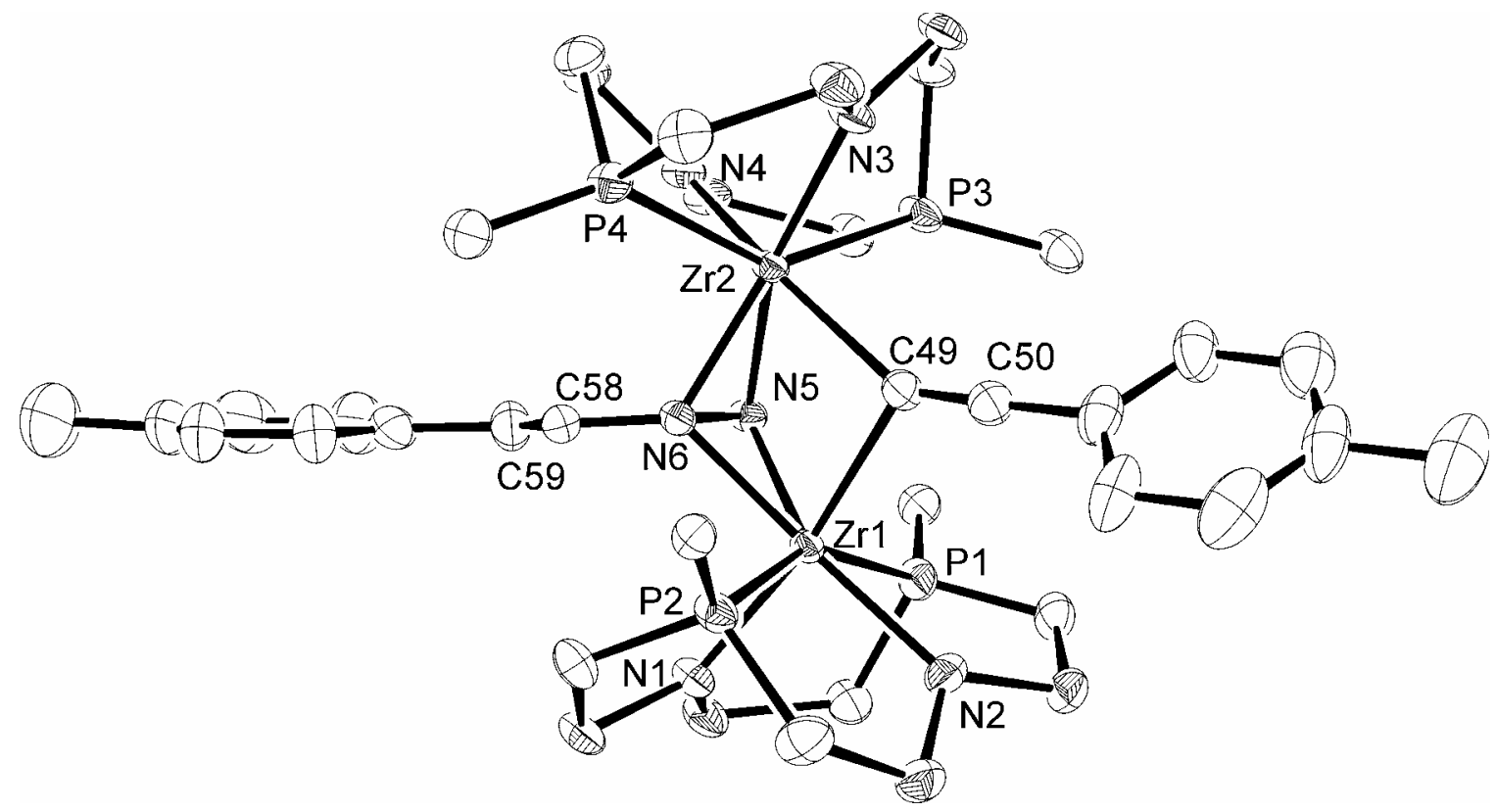

Figure 2: Molecular structure (ORTEP) of $\left(\left[\mathrm{P}_{2} \mathrm{~N}_{2}\right] \mathrm{Zr}\right)_{2}\left[\mu-\mathrm{N}_{2} \mathrm{CHCHC}_{6} \mathrm{H}_{4}\left(4-\mathrm{CH}_{3}\right)\right]\left[\mu-\mathrm{CCC}_{6} \mathrm{H}_{4}(4-\right.$ $\left.\mathrm{CH}_{3}\right)$ ], 3, determined by x-ray crystallography. Ellipsoids drawn 50\% probability level, silylmethyls are omitted for clarity and only ipso carbons of phenyl rings are shown.

Table 3: Selected Bond Lengths $(\AA)$ and Angles (deg) in $\left(\left[\mathrm{P}_{2} \mathrm{~N}_{2}\right] \mathrm{Zr}\right)_{2}\left[\mu-\mathrm{N}_{2} \mathrm{CHCHC} \mathrm{H}_{4}\left(4-\mathrm{CH}_{3}\right)\right][\mu-$ $\left.\mathrm{CCC}_{6} \mathrm{H}_{4}\left(4-\mathrm{CH}_{3}\right)\right](3)$

$\begin{array}{llll}\mathrm{N}(6)-\mathrm{N}(5) & 1.457(4) & \mathrm{N}(1)-\mathrm{Zr}(1)-\mathrm{N}(2) & 91.8(1) \\ \mathrm{N}(6)-\mathrm{C}(58) & 1.419(5) & \mathrm{N}(3)-\mathrm{Zr}(2)-\mathrm{N}(4) & 95.7(1) \\ \mathrm{C}(58)-\mathrm{C}(59) & 1.330(6) & \mathrm{P}(3)-\operatorname{Zr}(2)-\mathrm{P}(4) & 145.22(4) \\ \mathrm{C}(49)-\mathrm{C}(50) & 1.202(6) & \mathrm{P}(1)-\operatorname{Zr}(1)-\mathrm{P}(2) & 154.52(4) \\ \operatorname{Zr}(2)-\mathrm{C}(49) & 2.445(4) & \operatorname{Zr}(1)-\mathrm{C}(49)-\mathrm{Zr}(2) & 87.8(2) \\ \operatorname{Zr}(1)-\mathrm{C}(49) & 2.463(4) & \operatorname{Zr}(1)-\mathrm{N}(6)-\mathrm{Zr}(2) & 103.35(1) \\ \operatorname{Zr}(2)-\mathrm{N}(6) & 2.142(3) & \operatorname{Zr}(1)-\mathrm{N}(5)-\mathrm{Zr}(2) & 106.2(1) \\ \operatorname{Zr}(2)-\mathrm{N}(5) & 2.163(3) & & \\ \operatorname{Zr}(1)-\mathrm{N}(6) & 2.197(3) & & \\ \operatorname{Zr}(1)-\mathrm{N}(5) & 2.094(3) & & \\ \operatorname{Zr}(2)-\mathrm{P}(3) & 2.732(1) & \operatorname{Zr}(1)-\mathrm{P}(2) & 2.756(1) \\ \operatorname{Zr}(2)-\mathrm{P}(4) & 2.767(1) & \operatorname{Zr}(1)-\mathrm{P}(1) & 2.785(1) \\ \operatorname{Zr}(2)-\mathrm{N}(4) & 2.176(3) & \operatorname{Zr}(1)-\mathrm{N}(2) & 2.205(3) \\ \operatorname{Zr}(2)-\mathrm{N}(3) & 2.306(3) & \operatorname{Zr}(1)-\mathrm{N}(1) & \end{array}$




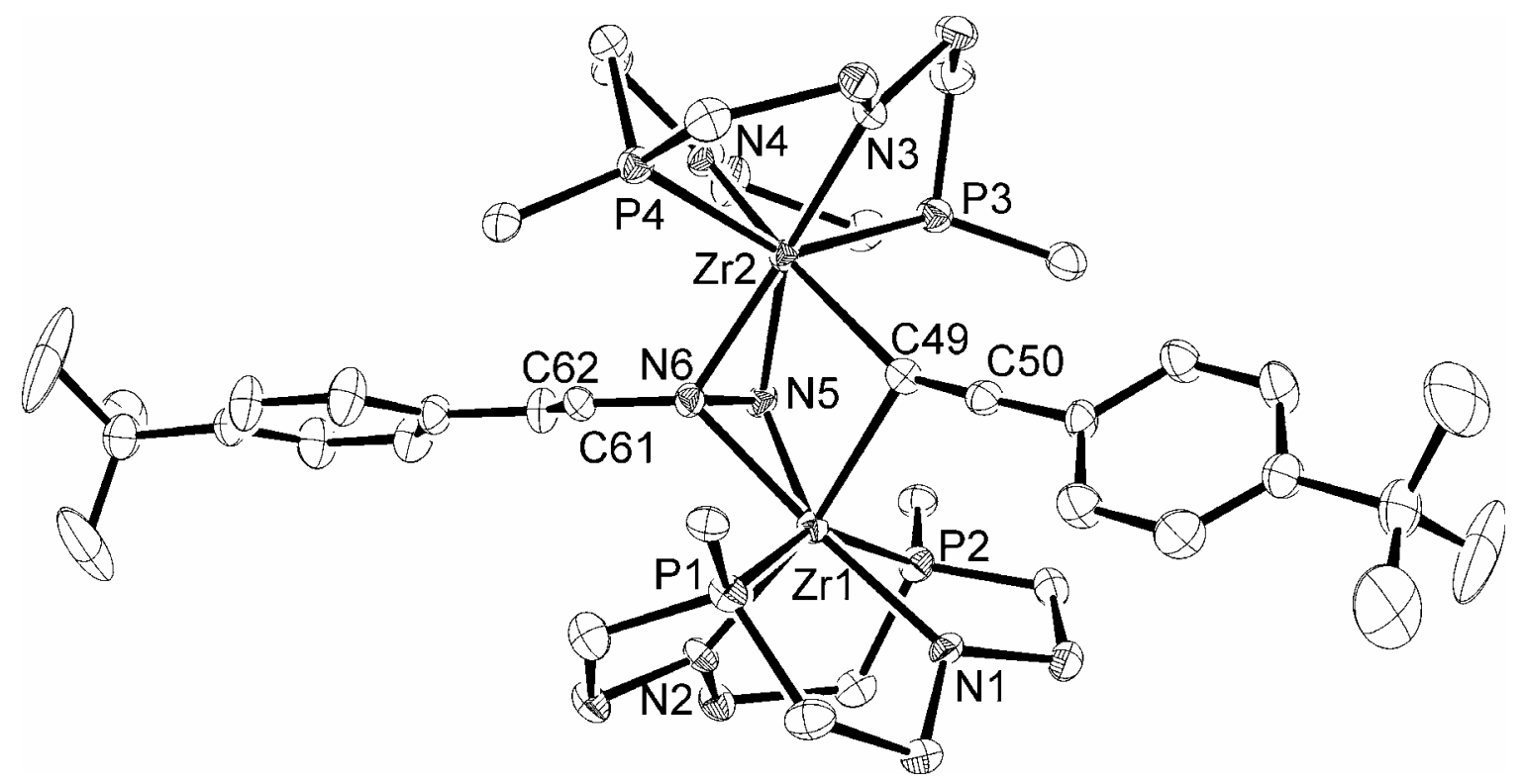

Figure 3: Molecular structure (ORTEP) of $\left(\left[\mathrm{P}_{2} \mathrm{~N}_{2}\right] \mathrm{Zr}\right)_{2}\left[\mu-\mathrm{N}_{2} \mathrm{CHCHC}_{6} \mathrm{H}_{4}\left(4-{ }^{\mathrm{t}} \mathrm{Bu}\right)\right]\left[\mu-\mathrm{CCC}_{6} \mathrm{H}_{4}(4-\right.$ $\left.{ }^{\mathrm{t}} \mathrm{Bu}\right)$ ], 4, determined by x-ray crystallography. Ellipsoids drawn 50\% probability level, silylmethyls are omitted for clarity and only ipso carbons of phenyl rings are shown.

Table 4: Selected Bond Lengths $(\AA)$ and Angles (deg) in $\left(\left[\mathrm{P}_{2} \mathrm{~N}_{2}\right] \mathrm{Zr}\right)_{2}\left[\mu-\mathrm{N}_{2} \mathrm{CHCHC} \mathrm{H}_{4}\left(4-{ }^{\mathrm{t}} \mathrm{Bu}\right)\right][\mu-$ $\left.\mathrm{CCC}_{6} \mathrm{H}_{4}\left(4-{ }^{\mathrm{t}} \mathrm{Bu}\right)\right]$ (4)

$\begin{array}{llll}\mathrm{N}(6)-\mathrm{N}(5) & 1.454(4) & \mathrm{N}(1)-\operatorname{Zr}(1)-\mathrm{N}(2) & 92.0(1) \\ \mathrm{N}(6)-\mathrm{C}(61) & 1.416(4) & \mathrm{N}(3)-\operatorname{Zr}(2)-\mathrm{N}(4) & 95.2(1) \\ \mathrm{C}(62)-\mathrm{C}(61) & 1.327(5) & \mathrm{P}(3)-\operatorname{Zr}(2)-\mathrm{P}(4) & 145.71(3) \\ \mathrm{C}(49)-\mathrm{C}(50) & 1.223(5) & \mathrm{P}(1)-\mathrm{Zr}(1)-\mathrm{P}(2) & 152.52(3) \\ \operatorname{Zr}(2)-\mathrm{C}(49) & 2.446(4) & \mathrm{Zr}(1)-\mathrm{C}(49)-\operatorname{Zr}(2) & 87.7(1) \\ \operatorname{Zr}(1)-\mathrm{C}(49) & 2.473(3) & \operatorname{Zr}(1)-\mathrm{N}(6)-\operatorname{Zr}(2) & 102.6(1) \\ \operatorname{Zr}(2)-\mathrm{N}(6) & 2.161(3) & \operatorname{Zr}(1)-\mathrm{N}(5)-\operatorname{Zr}(2) & 107.7(1) \\ \operatorname{Zr}(2)-\mathrm{N}(5) & 2.144(3) & & \\ \operatorname{Zr}(1)-\mathrm{N}(6) & 2.206(3) & & \\ \operatorname{Zr}(1)-\mathrm{N}(5) & 2.076(3) & & \\ \operatorname{Zr}(2)-\mathrm{P}(3) & 2.736(1) & \operatorname{Zr}(1)-\mathrm{P}(1) & 2.769(1) \\ \operatorname{Zr}(2)-\mathrm{P}(4) & 2.756(1) & \operatorname{Zr}(1)-\mathrm{P}(2) & 2.7780(9) \\ \operatorname{Zr}(2)-\mathrm{N}(4) & 2.188(3) & \operatorname{Zr}(1)-\mathrm{N}(1) & 2.214(3) \\ \operatorname{Zr}(2)-\mathrm{N}(3) & 2.303(3) & \operatorname{Zr}(1)-\mathrm{N}(2) & \end{array}$




\section{References}

1) Fryzuk, M. D.; Love, J. B.; Rettig, S. J.; Young, V. G. Science 1997, 275, 1445-1447.

2) Beurskens, P. T.; Admiraal, G.; Beurskens, G.; Bosman, W. P.; Garcia-Granda, S.; Gould, R. O.; Smits, J. M. M. PATTY; The DIRDIF program system, Technical Report of the Crystallography Laboratory: University of Nijmegen, The Netherlands, 1992.

3) Beurskens, P. T.; Admiraal, G.; Beurskens, G.; Bosman, W. P.; Gelder, R. d.; Isreal, R.; Smits, J. M. M. DIRDIF94; The DIRDIF-94 program system, Technical Report of the Crystallography Laboratory: University of Nijmegen, The Netherlands, 1994.

4) $d^{*}$ TREK; Area Detector Software. Version 4.13. Molecular Structure Corporation, 1996-1998.

5) Altomare, A.; Cascarano, M.; Giacovazzo, C.; Guagliara, A. J. Appl. Cryst. 1994, 26, 343.

6) Altomare, A.; Burla, M. C.; Cammalli, G.; Cascarano, M.; Giacovazzo, C.; Guagliardi, A.; Moliterni, A. G. G.; Polidori, G.; Spagna, A. J. Appl. Cryst. 1999, 32, 115-119. 